\title{
Emergent Technologies for Active Aging
}

\author{
Thais Andrea Baldissera ${ }^{1}$, Cristiano De Faveri ${ }^{2}$ \\ ${ }^{1}$ Electrical and Computer Engineering - FCT \\ Universidade NOVA de Lisboa \\ ${ }^{2}$ NOVA LINCS, Department of Computer Science \\ Faculty of Science and Technology \\ Universidade NOVA de Lisboa \\ t.baldissera, c.faveri\{@campus.fct.unl.pt $\}$
}

\begin{abstract}
Continuous advances in medicine and wellness areas have contributed to an active and enjoyable aging. The use of technology in assisting elderly community is expected to have a positive contribution to support the diversity of required services that promote independent living. Most initiatives in this area are intrinsically focused on providing health-care services to senior citizens. However, a broader perspective of the aging process is required, which is conceptually described as "active aging". In this paper, we present some prominent technologies based on context-awareness and Internet of Things that may assist elderly people in different needs during a productive live. We exemplify the applicability of such technologies by describing a scenario in which daily activities are performed by an elderly assisted by her son.
\end{abstract}

\section{Introduction}

In the last century, one of the most important demographic changes in the history of humanity has taken place: the aging of the global population. Current trends [Forum 2013, Fengler 2014, Gartner 2014] suggest that elderly population will surpass young people in many regions of the world. This change is already consolidated today in countries such as Portugal, where the population over 60 years is $10 \%$ higher than people under 15 years old, which was inversely proportional 30 years ago. By 2050, it is expected that population over 80 years exceeds the young [HelpAgeInternational 2014].

Current estimates indicate that $60 \%$ of elderly people live by themselves or in the company of another elderly person, and strive to guarantee their autonomy and perform their own duties. With continuous advances in medicine and wellness areas, people aspire to an active and enjoyable aging [Forum 2013] although aging might as well entail several limitations due to impairment. In extreme cases, regular daily activities such as cooking, personal hygiene, housework's, etc., are often affected [Spar and La Rue 2007].

The traditional way to deal with this problem requires intense care from family. Relatives need to actively participate in the aging process and support elderly. In this case, many people live with their family, specially when they begin to loose capabilities. Those who remain living by themselves either, care for themselves alone or require caregivers to assist them on daily activities. Alternatively, the elderly stays in a nursing home. All these changes, can directly affect the senior life style [Spar and La Rue 2007]. The experience 
of aging can make people more demanding and grumpy, as they may not like this new lifestyle, or find that they are considered a burden to the family, having depression and sadness moments. Nevertheless, it is a process that requires dedication (attention care from family) and financial resources.

The use of technology in assisting the elderly community is expected to have a positive contribution to overcome identified challenges and supporting a diversity of the required services to keep independent living [Alwan et al. 2007, Czaja and Lee 2009, Camarinha-Matos et al. 2013a, Forum 2013]. However most elderly people still face several barriers in terms of inclusion in an increasingly technological society. This fact puts older adults at a disadvantage, requiring that researchers and practitioners deliver more suitable products for them [Spar and La Rue 2007, Czaja and Lee 2009, Cresci et al. 2010].

Some examples of technological solutions include smart devices, like wearable devices, sensors and associated software services that provide real-time data about an individual and the environment he/she lives in; a bracelet that uses a GPS sensor to set up a "safe zone" for people with cognitive disorders; an at-home patient-tracking device with a panic button for emergencies; and cameras and sensors that capture movements, calculate body weight and make the measurement of heart-bit rate [Scoble and Israel 2014].

The adoption of context-aware analysis techniques, combined with smart devices and sensors, are likely to enable a great number of new services without much effort in usage. In fact, it may be expectable that whith increased intelligence in the new systems, interaction with technology becomes smoother and gradually becomes part of daily life for seniors, family members, caregivers and integrated service providers.

In this paper, we present some prominent context-aware technologies that can be applied to assist elderly people in different areas of life. Global trends about aging (Section 2) settle the opportunities for new technologies for elderly. In particular, we present context-awareness technologies (Section 3) as part of a major scenario integrating the Internet of Things (Section 4). We then present a story line about the routine of an elderly person and discuss elements for a possible service model (Section 5). Finally, we conclude (Section 6) with a discussion about the main advantages and concerns on emergent technologies for elderly community.

\section{Background Review}

Current trends [Forum 2013, Fengler 2014, Gartner 2014] suggest that the percentage of elderly population is increasing significantly. In continents such as Europe, the current proportion of elderly is around $24 \%$ of the population, corresponding approximately to 175 millions of people while young people represent around 117 million (16\%). In 2050, although the Europe's population tends to decrease, the number of seniors is expected to reach $27.2 \%$ of the population in Europe [Gartner 2014].

Figure 1 shows the total fertility rate in various continents, comparing the situation in 1970 with 2014. In 1970, each woman had 4.7 children on average in the world, while in 2014, this number decreases to 2.5. The same trend can be observed in all continents, reaching a dramatic situation in the case of Europe and North America.

Once an adult reaches the age of 60 , she/he can expect, on average, 13 years more 


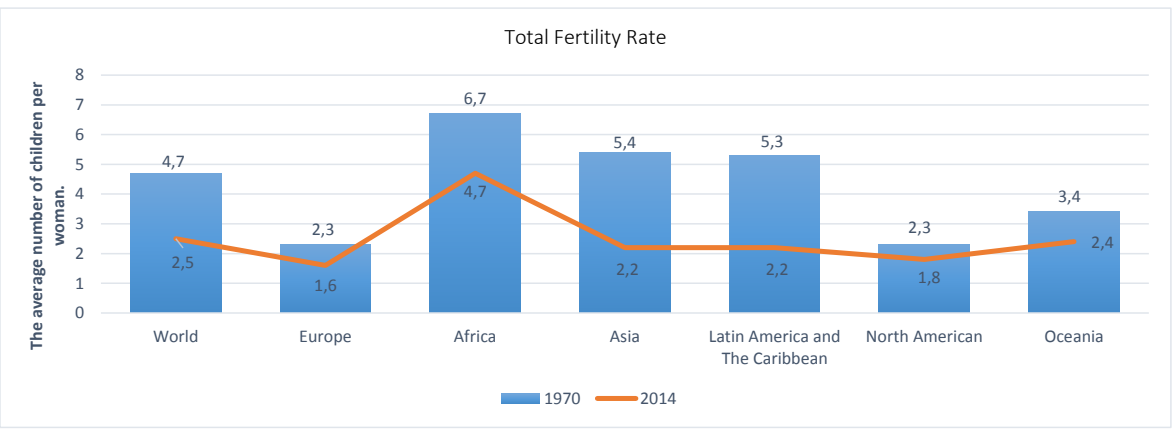

Figure 1. Total Fertility Rate in the World and Continents. Adapted from (http://www.prb.org/wpds/2014/, 2014)

of full healthy life [Organization 2010, Chiarini et al. 2013]. This trend can be observed in Figure 2, where the life expectancy in the world has grown 22\% (13 years) from 1970 to 2014. In all continents, this situation is similar. This century people are more active and healthier into their 70s and 80s, travelling abroad, dining out, and spending a higher proportion of their incomes on food and drink than those under 60 [Cresci et al. 2010, Enterprise 2015].

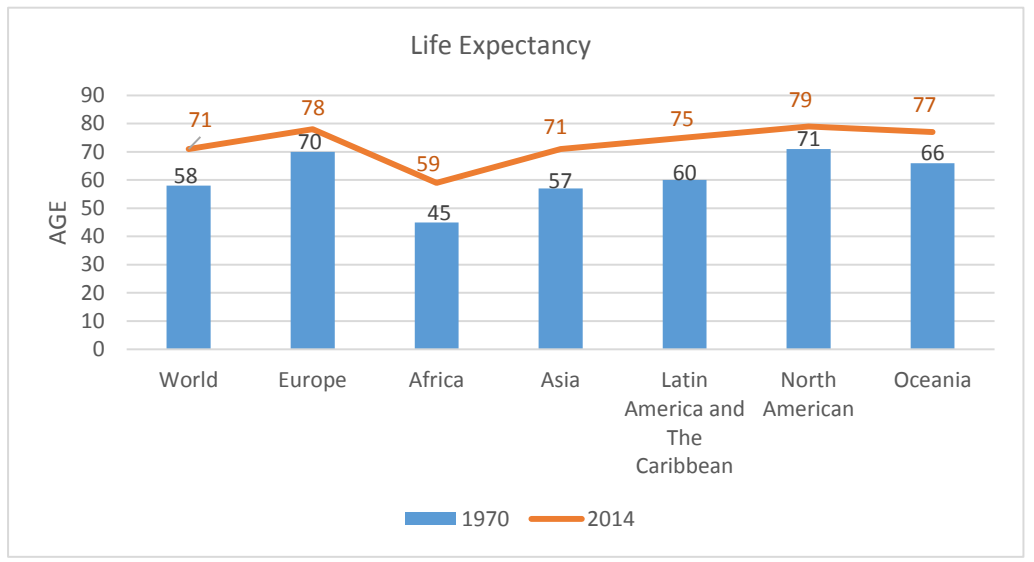

Figure 2. Life Expectancy in the World and the Continents. Adapted from (http://www.prb.org/wpds/2014/, 2014)

In the case of Brazil, the young population is still higher than the senior population. However, trends show a decline of young people and a growing of older population, as presented in Figure 3. Seniors group (60 - 79 and 80+) grows up while all others have decline.

Being an inevitable and widespread phenomenon around the world, the growth of elderly population imposes structural changes in society, with impacts on the economic growth, on the labor market, on the capital and consumption, on health systems and social support, and chiefly on family structures.

Aging can also cause many governmental changes. These amendments have implications on many areas of policy and practice, including that of $\mathrm{ICT}^{1}$ systems. These

\footnotetext{
${ }^{1}$ Information and Communication Technology
} 


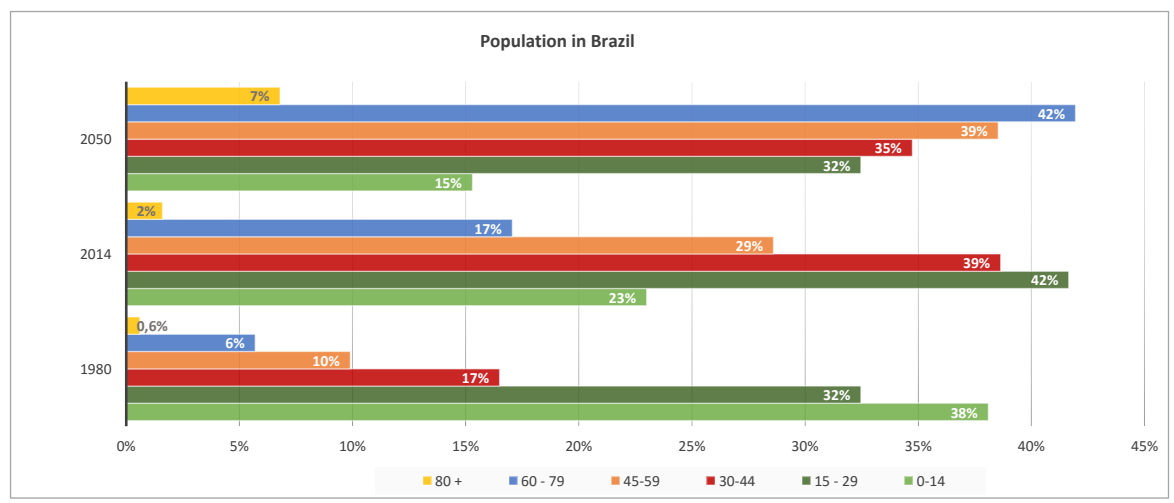

Figure 3. Population's Graphic in Brazil 1980, 2014 and 2050.Based in United Nations, Department of Economic and Social Affairs, Population Division. World Population Prospects: The 2012 Revision and Global AgeWatch Index 2014.

systems are becoming more and more part of daily life, presenting valuable opportunities for supporting and engaging older people. ICT and assistive technologies, in general, have become an integrated part in the life of many people, including the elderly.

\section{Context-Aware Technologies}

A context represents any information that can be used to characterize the situation of an entity [Abowd et al. 1999, Dey et al. 2001, Yau and Karim 2004]. An entity is a person, place, or object that is considered relevant to the interaction between a user and an application, including location, time, activities, and the preferences of each entity. The concept of context can be described independently of the actions done, but some authors [Dourish 2001, Winograd 2001] understand that something is contextualized because of the way it is used in interpretation. On the other hand, [Sato 2003] proposes to represent context through "a pattern of behavior or relations among variables that are outside of the subjects of design manipulation and potentially affect user behavior and system performance".

Context awareness is about capturing a broad range of contextual attributes (such as the user's current position, activities, and their surrounding environment) to better understand what the user is trying to accomplish, and what services the user might be interested in [Lee 2007]. For [Costa 2003] context-awareness seeks to exploit humancomputer interactions by providing computing devices with knowledge of the users' environment, i.e., with context. Context awareness computing is the most recent development in the progression of technology. It refers to the fact that technologies are starting to "understand" things about you and your environment [Scoble and Israel 2014].

Currently, many technologies have contributed to the development of contextaware solutions. They have enlarged the possibilities of producing cutting-edge products dedicated to distinct segments of our society. In a broad sense, [Scoble and Israel 2014] highlight five forces that have shaped and cooperated with the advances on context-aware technologies. We briefly describe these forces as follows:

- Mobile: [CISCO 2014] shows that active mobile devices already outnumbered the number of people on Earth in 2012. Wearable computing is booming, estimating 
the unit sales of wearable's will rise from 15 million in 2013 to 70 million by 2017 [Research 2014], data costs are dropping [Research 2014], and application downloads are a massive practice.

- Social Media: Investments on social networks will increase by $300 \%$ over the next 5 years, a growth fuelled by the desire of individual and organizations to distribute timely content with their community [Forrester 2015]. More than 2 billion people are on social networks, and businesses are using them to be connected with customers, to offer specialized services and learn from their experience. Segmented social-networks has loomed for distinct interests. In particular, social networks for elderly has emerged as an opportunity to maintain social relationships, touch with friends and family, and find specific services.

- Data: The size of the Internet is expanding at an exponential rate - leading to the idea of big data. It is estimated that $90 \%$ of the world's data was created in the last two years [Gartner 2014]. The demand for analytics provides a level of quantitative analysis on key data sets impacting a business and more importantly, where informed decisions can be taken. By identifying a new standard in the elderly context, for example, when seniors stop working (possibly retired), specific services related to occupancy in life may have more relevance and can be offered during their new life phase.

- Sensors: Sensors in technology can emulate three of the five human senses: sight, touch, and hearing. For instance, sensors for temperature, humidity provide the necessary data to automatically adjust the comfort level and to optimize the use of energy for heating or cooling (ubiquitous sensor networks) or smart metering for measuring energy consumption and transmitting this information to the energy provider electronically.

- Location-based services: Our location is one of the most important parts of our context because it can transform some key characteristics of customer interaction, such the scope of the service, and its duration and frequency [Fano and Gershman 2002].

These forces can directly influence decisions through a context analysis and a wide kind of sensors, smart devices and services compounded by new technologies. These devices in a communicating-actuating network creates the Internet-of-Things (IoT), where sensors and actuators blend seamlessly with the environment around us, and the information is shared across platforms in order to develop a common operating picture.

\section{Internet of Things}

The term IoT typically refers to the interconnection of uniquely identifiable embedded computing devices within existing Internet infrastructure. The term is closely related to smart objects that interact and communicate with other machines, physical environments, and infra structures [Choi 2014]. Applications of IoT have been investigated in a number of domains such as smart cities, intelligent buildings, health, smart grids, logistic and transportation, and agriculture and animal farming.

IoT is an integrated part of Future Internet and could be defined as a dynamic global network infrastructure with self-configuring capabilities based on standard and interoperable communication protocols where physical and virtual 'Things' have identities, 
physical attributes, virtual personalities and use intelligent interfaces, seamlessly integrated into the information network [Sundmaeker et al. 2010]. A 'Thing' in this context could be defined as a real/physical or digital/virtual entity that exists and moves in space and time and is capable of being identified. Things are commonly identified either by assigned identification numbers, names and/or location addresses. This interpretation is shared by [Camarinha-Matos et al. 2013b]. According to these authors, Cyber Physical Systems includes not only 'Things' connected to the Internet, but also other physical systems with embedded computational power.

In a high-level perspective, the Internet of Things requires 3 major components [Gubbi et al. 2013]: (i) hardware - sensors, actuators and embedded communication hardware, (ii) middleware - data storage and tools for data analytics, and (iii) presentation - visualization and interpretation tools widely accessed on different platforms for different applications. We describe a few enabling technologies which coop to make up these components.

- Radio Frequency Identification (RFID): corresponds to the cornerstone of embedded wireless data communication technologies. A RFID tag is able to store, collect and transmit information about a particular item, providing a unique ID for the object, acting as an electronic barcode [Welbourne et al. 2009, Juels 2006]. Passive RFID tags are battery-free and obtain energy from the reader's interrogation signal to communicate the ID to the RFID reader. On the other hand, active RFID tags are powered by an internal battery used to run the internal microchip's circuit and to broadcast a signal to readers. Current applications of RFID technology can be found in a number of domains such as transportation, access control, bank cards and toll tags.

- Wireless Sensor Networks (WSN): this network is compounded by a large number of intelligent sensors, which collect, process, analyze and disseminate valuable information gathered in a variety environments [Akyildiz et al. 2002]. Active RFID close represents the lower end WSN nodes with limited processing capability and storage. Sensor data are shared among sensor nodes and sent to a system for analytics. The components of WSN include hardware containing sensor interfaces, processing units, transceiver units and power supply; communication stack to enable robust data transmission between sensors and the outside world through the Internet; WSN middleware that combines cyber infrastructure, service-oriented architectures (SOA) and sensor networks to enable access to heterogeneous sensor resources; and secure data aggregation that implements secure and efficient mechanisms on sensor nodes to protect the systems from adversaries.

- Addressing schemes: the ability to uniquely identify 'Things' is crucial for the success of IoT. Every element already connected and those that are going to be connected must be identified by their unique identification, location and capabilities. Moreover, the addition of networks and devices must not hamper the functional aspects of the devices, performance and reliability of the network. In this sense, initiatives like IPv6 have collaborated with the solution of the problem of uniquely identify resources.

- Data storage and analytics: with the large amount of data produced by IoT devices, issues like storage, ownership and expiration must be addressed. Moreover, automated decision making and fusion algorithms are required to process data effi- 
ciently in a centralized infrastructure to support storage and analytics. This is part of the IoT middleware layer, which a number of challenges have been debated. A comprehensive discussion about these issues can be found on [Gubbi et al. 2013].

- Presenting : attractive and usable visualization is important to take full advantage of the entire potential IoT may offer to individuals. Recent advances in touch screen technologies have enabled the use of smart tablets and phones has become very intuitive. Also, 3D technologies provide more meaningful ways to present information for consumers. With the huge amount of information sensors can generate at fast pace, visualization technologies represent another important concern of IoT.

[Global 2013] suggests that IoT is the next revolution in computing. While smartphones and mobile Internet enabled the advent of applications for the consumer, the future induces to the expectation of pervasive integration of semiconductors, mobile communication, and big data/analytics propelling the Internet of Things into the wider economy.

\section{ICT Scenario for EIderly}

In order to better illustrate ICT solutions applied on elderly care, a scenario with our fictitious characters Lucy and her son Marco is presented.

"Mrs Lucy is a 70 years old and very active lady. She likes to go out with friends, attend social events, and do social work. She is retired, still performing some occupations. She lives with her son Marco who subscribed to the collaborative model services from the care center of his town. These services provide daily life support for his mother in the several contexts, inside and outside home. There are several sensors which help monitoring her safety and well-being. When going out, Mrs Lucy uses to use low-cost wearable sensors, which are used to monitor her well-being and tracks her localization. When necessary, these sensors interact with her smart phone, to send relevant information to the care center. One of these days, she planned to meet her friend Sarah and visit the science museum. The itinerary includes walking and taking public transportation. The GPS service running in her phone helps her to plan the trip. It displays her location and indications to move to the intermediate locations of the itinerary, and to get in the public transports. Another service, personal activity management, uses the itinerary information to schedule the events for the corresponding depart time, assisting Mrs Lucy to remember the appropriate timing. When she takes the bus, the destiny recorded in her smart device synchronizes with the bus route. Mrs Lucy arrives at the museum and meets Sarah. When she gets inside the museum, her smart phone switches to the museum's internal map and localization system. The map displays multi-floor visual representations of the museum, showing the location of the rooms containing several thematic items they might want to see. But firstly, the friends feel like having a coffee, so its location was pinpointed in the map. While going to the coffee, the smart phone would display graphic or audio messages indicating the directions to be taken, e.g. "move forward", "turn left", "go upstairs", etc. Mrs Lucy comes back home really tired so she decides to sit awhile to watch TV. She receives daily pillbox notification that she needs to take them punctually at 9. However, she decides to stay a little bit longer, finish watching her favorite soap opera. In the meantime, she sleeps. At 9:10 she receives a call on her smart phone from her virtual assistant to take her medication. When she takes the medication, the pillbox sends to her son Marco the confirmation. In the morning also, the smart bed sends to him the mother's 
sleep report. He is satisfied and unworried because she didn't have any sleepwalking episode and the emergency service was not activated. Another day, Lucy and her friend Antony decided to attend a concert in another city. The alarm rings 30 minutes ahead of schedule, as it is the eve of a holiday. The public transport is delayed and Mrs Lucy needs more comminuting than usual. The itinerary requires walking, going by bus to a train station, taking a long train course to the city, and finally getting a bus to the concert-hall. The first step is to go to the nearest station to buy the travel tickets. She approaches the ticket machine with the itinerary showing in the smart phone's screen. Using her finger, her wearable clothes identify the movements and its "throws" the itinerary in the direction of the machine. Using the information regarding the itinerary, the ticket machine prints a multi-modal, RFID-based ticket that is good for several transport systems used to reach the concert hall. The cost is automatically deduced from her budget plafond-customized credit card account. As before, the relevant events, such as depart time and so on, are also handled by her daily activity management service. Last month, Lucy felt very bad and felt down on the street. Her clothing identified the falling and started a calling to the emergency service, which her medical data already sent by the smart device. Fortunately it was nothing serious and his son didn't have to move the work. While outside home, the wearable sensors constantly monitor her heart rate, body temperature, localization, and so on, which are used by a service running in her smart phone to assert her well-being. At home, smart appliances also perform the work of assisting Mrs Lucy and Marco. The fridge reads the RFID tags of the several items that it has got inside, suggest discards and notify reaching expiration date. With this information, and also analyzing Mrs Lucy's consuming behavior and health, the fridge adds items to the shopping list managed by the smart phone. Mrs Lucy can decide to go for shopping, which an itinerary including the location of the best-price stores or she can wait for the products on her house.

From this illustrating scenario, we can identify many aspects that are relevant for the specification of a model that supports a set of needs and subsequent services for the required particularities for aging. We highlight some of these aspects as follows:

- There are several entities present in the story: elders/users, families, friends, monitoring sensors, home locations.

- There are several services providing assistance to Mrs. Lucy, both inside and outside home, e.g. personal activity management, well-being monitoring, medication control, GPS itinerary and localization, inside building location, integrated public transportation services, and so on. These are services that the model must support.

- The smart appliances inside home are self-managed and interconnected. In elderly home, some food in the fridge might be fully consumed or expire their validation. Such packages of items like milk, yogurts, sauce, and so on, come with RFID tags, which transmit their characteristics to the fridge unit. The fridge warns the elderly to take out the expired items. These can be considered home services. When the elderly goes to the market, its shopping list is updated with the needs for the finished or expired food. If food items are from distinct stores, the GPS application prepares the adequate itinerary for shopping them. The IoT pillbox warns that the pill was not taken and interacts with the personal activity management. The wearable clothes identify movements and transmit data to the appropriate iterations: Call emergency or ticket machine. This illustrates the need for integration or combination of services. 
- The mentioned GPS application, running in the elder's smart device, to be personalized for elderly people, allowing them to walk safely through the city. This application would track the location and itinerary of these people, as they are going to e.g. visit friend's homes, going to coffee shops, to the shops/supermarkets, bus stations, museums, concert halls, and so on. These places would automatically be saved as regular destinations, from which the users could easily select next times for determining corresponding itinerary. This is an example of the tailoring services for elder people based on context aware (location). These services are managed and updated in real time (e.g. public transportation schedule based, the elderly travel speed based, elderly fall).

- Buses could have wireless electronic identification (WEI), which can broadcast to passengers smart devices inside the bus. With this identification, smart devices can receive the itinerary, bus stops, and interconnections with other buses or other public transports. The information on these interconnections are achieved because the corresponding companies collaboratively participate to provide such integrated support. This is another example of the tailoring GPS collaborative services for elder people though multi-providers.

- In Mrs. Lucy's scenario, she gets inside the bus, her smart phone receives its WEI, allowing the GPS pre-planned itinerary be synchronized with the bus itinerary. Setting the alarm time was accomplished by providing the traffic situation at the time. This could be part of previous service.

- When Mrs. Lucy enters into the museum, which has also got a WEI. The smart phone receives the museum map with the location of thematic objects to be seen in the several rooms. Devices across the museum provide localization data to the smart phone. Lucy presses on the screen the desired location in the museum, through an adjusted and intuitive GUI. The smart device would indicate the directions towards the desired location. This is another example of a service (inbuilding itinerary and localization).

- Similarly to the museums, other public or private buildings, such as railway stations, shopping/malls may also have got WEI with localization functionality. These are services similar to the previous one, but provided by other service providers.

- IoT pillbox, wearable clothes, smart mobile phone, smart shoes, smart bed, tickets machines, bus interconnected are devices networks. These are IoT devices on pervasive and mobile computing environment adapted to the needs of seniors, caregivers, family and service providers.

\section{Conclusions}

In the era of IoT, many services will promote the extension of capabilities of elderly people for an active aging. In this paper we highlight some prominent technologies to support an active and productive aging, as new perspectives for the concept of being elderly. The adoption of context-aware analysis techniques, combined with smart devices and sensors, will enable a great number of seamless and personalized services. As the intelligence in new systems grows, the technology becomes more interactive and gradually will be part of daily life for seniors, family members, caregivers and integrated service providers. While envisioned as a revolution on services, it is important to recognize that the economic growth alone will not improve older people's well-being and specific policies are 
required to address the implications of aging. Many computer tasks are still characterized by having high cognitive demands and, for this reason, it is important to hand any age-related changes on cognitive skills, when designing systems that target older adults. Because of the importance of improving the management and delivery of health care and social services, ICT plays a key role on developing quality of life of older people. Nevertheless, such initiatives are expected to assist on the mitigation of situations of isolation and loneliness, and improve family and community relationships.

\section{Acknowledgments}

This work is funded by Ciências sem Fronteiras / CAPES Foundation / Brazil.

\section{References}

Abowd, G. D., Dey, A. K., Brown, P. J., Davies, N., Smith, M., and Steggles, P. (1999). Towards a better understanding of context and context-awareness. In Handheld and ubiquitous computing, pages 304-307. Springer.

Akyildiz, I. F., Su, W., Sankarasubramaniam, Y., and Cayirci, E. (2002). Wireless sensor networks: a survey. Computer networks, 38(4):393-422.

Alwan, M., Wiley, D., and Nobel, J. (2007). State of technology in aging services. Center for Aging Services Technology (CAST).

Camarinha-Matos, L. M., Afsarmanesh, H., Ferrada, F., Oliveira, A. I., and Rosas, J. (2013a). A comprehensive research roadmap for ict and ageing. Studies in Informatics and Control, 22(3):233-254.

Camarinha-Matos, L. M., Goes, J., Gomes, L., and Martins, J. (2013b). Contributing to the Internet of Things, pages 3-12. Springer.

Chiarini, G., Ray, P., Akter, S., Masella, C., and Ganz, A. (2013). mhealth technologies for chronic diseases and elders: A systematic review. Selected Areas in Communications, IEEE Journal on, 31(9):6-18.

Choi, A. J. (2014). Internet of things: Evolution towards a hyper-connected society. In Solid-State Circuits Conference (A-SSCC), 2014 IEEE Asian, pages 5-8. IEEE.

CISCO, C. V. N. (2014). Global mobile data traffic forecast update, 2014-2019 white paper.

Costa, P. D. (2003). Towards a services platform for context-aware applications. Telematics, University of Twente, Enschede. Netherlands. August.

Cresci, M. K., Yarandi, H. N., and Morrell, R. W. (2010). Pro-nets versus no-nets: Differences in urban older adults' predilections for internet use. Educational Gerontology, 36(6):500-520.

Czaja, S. J. and Lee, C. C. (2009). Information technology and older adults. CRC Press.

Dey, A. K., Abowd, G. D., and Salber, D. (2001). A conceptual framework and a toolkit for supporting the rapid prototyping of context-aware applications. Human-computer interaction, 16(2):97-166.

Dourish, P. (2001). Seeking a foundation for context-aware computing. Human-Computer Interaction, 16(2-4):229-241. 
Enterprise, M. B. F. (2015). Idosos estão consumindo mais.

Fano, A. and Gershman, A. (2002). The future of business services in the age of ubiquitous computing. Commun. ACM, 45(12):83-87.

Fengler, W. (2014). The end of the population pyramid. The World Bank.

Forrester, C. (2015). The next app platforms will bridge corporate, consumer, commerce, and connected devices. Report.

Forum, T. C. G. (2013). Understanding the needs and consequences of the ageing consumer.

Gartner, I. (2014). Applying lessons from 20 years of hype cycles to your own innovation and forecasting strategies. Report.

Global, M. S. R. (2013). Technology - the internet of things is now. Report.

Gubbi, J., Buyya, R., Marusic, S., and Palaniswami, M. (2013). Internet of things (iot): A vision, architectural elements, and future directions. Future Generation Computer Systems, 29(7):1645-1660.

HelpAgeInternational (2014). Global agewatch index 2014. Report.

Juels, A. (2006). Rfid security and privacy: A research survey. Selected Areas in Communications, IEEE Journal on, 24(2):381-394.

Lee, W.-P. (2007). Deploying personalized mobile services in an agent-based environment. Expert Systems with Applications, 32(4):1194-1207.

Organization, W. H. (2010). World health statistics 2010. World Health Organization.

Research, J. (2014). Smart wearable devices: Market trends \& competitive landscape 2014-2019. Report.

Sato, K. (2003). Context sensitive interactive systems design: A framework for representation of contexts. In Proceedings of the 10th International Conference on $\mathrm{Hu}$ man-Computer Interaction, volume 3, pages 1323-1327. Citeseer.

Scoble, R. and Israel, S. (2014). Age of Context: mobile, sensors, data and the future of privacy.

Spar, J. E. and La Rue, A. (2007). Clinical manual of geriatric psychiatry. American Psychiatric Pub.

Sundmaeker, H., Guillemin, P., Friess, P., and Woelfflé, S. (2010). Vision and challenges for realising the internet of things.

Welbourne, E., Battle, L., Cole, G., Gould, K., Rector, K., Raymer, S., Balazinska, M., and Borriello, G. (2009). Building the internet of things using rfid: the rfid ecosystem experience. Internet Computing, IEEE, 13(3):48-55.

Winograd, T. (2001). Architectures for context. Human-Computer Interaction, 16(2):401-419.

Yau, S. S. and Karim, F. (2004). An adaptive middleware for context-sensitive communications for real-time applications in ubiquitous computing environments. Real-Time Systems, 26(1):29-61. 